\title{
Front Matter: Volume 10576
}

, "Front Matter: Volume 10576," Proc. SPIE 10576, Medical Imaging 2018: Image-Guided Procedures, Robotic Interventions, and Modeling, 1057601 (2 July 2018); doi: 10.1117/12.2323924

SPIE. Event: SPIE Medical Imaging, 2018, Houston, Texas, United States 


\title{
PROGRESS IN BIOMEDICAL OPTICS AND IMAGING
}

\section{Medical Imaging 2018}

\section{Image-Guided Procedures, Robotic Interventions, and Modeling}

\author{
Baowei Fei \\ Robert J. Webster III
}

12-15 February 2018

Houston, Texas, United States

Sponsored by

SPIE

Co-sponsored by

DECTRIS Ltd. (Switzerland)

Cooperating Organizations

AAPM-American Association of Physicists in Medicine (United States)

IFCARS - International Foundation for Computer Assisted Radiology and Surgery (Germany)

MIPS-Medical Image Perception Society (United States)

RSNA-Radiological Society of North America (United States)

WMIS—World Molecular Imaging Society

Published by

SPIE

Volume 10576 
The papers in this volume were part of the technical conference cited on the cover and title page. Papers were selected and subject to review by the editors and conference program committee. Some conference presentations may not be available for publication. Additional papers and presentation recordings may be available online in the SPIE Digital Library at SPIEDigitalLibrary.org.

The papers reflect the work and thoughts of the authors and are published herein as submitted. The publisher is not responsible for the validity of the information or for any outcomes resulting from reliance thereon.

Please use the following format to cite material from these proceedings:

Author(s), "Title of Paper," in Medical Imaging 2018: Image-Guided Procedures, Robotic Interventions, and Modeling, edited by Baowei Fei, Robert J. Webster III, Proceedings of SPIE Vol. 10576 (SPIE, Bellingham, WA, 2018) Seven-digit Article CID Number.

ISSN: 1605-7422

ISSN: 2410-9045 (electronic)

ISBN: 9781510616417

ISBN: 9781510616424 (electronic)

Published by

SPIE

P.O. Box 10, Bellingham, Washington 98227-0010 USA

Telephone +1 3606763290 (Pacific Time) · Fax +1 3606471445

SPIE.org

Copyright (C) 2018, Society of Photo-Optical Instrumentation Engineers.

Copying of material in this book for internal or personal use, or for the internal or personal use of specific clients, beyond the fair use provisions granted by the U.S. Copyright Law is authorized by SPIE subject to payment of copying fees. The Transactional Reporting Service base fee for this volume is $\$ 18.00$ per article (or portion thereof), which should be paid directly to the Copyright Clearance Center (CCC), 222 Rosewood Drive, Danvers, MA 01923. Payment may also be made electronically through CCC Online at copyright.com. Other copying for republication, resale, advertising or promotion, or any form of systematic or multiple reproduction of any material in this book is prohibited except with permission in writing from the publisher. The CCC fee code is 1605$7422 / 18 / \$ 18.00$.

Printed in the United States of America.

Publication of record for individual papers is online in the SPIE Digital Library.

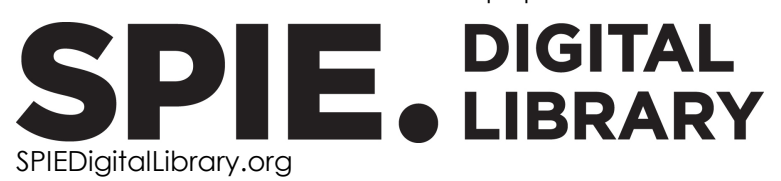

Paper Numbering: Proceedings of SPIE follow an e-First publication model. A unique citation identifier (CID) number is assigned to each article at the time of publication. Utilization of CIDs allows articles to be fully citable as soon as they are published online, and connects the same identifier to all online and print versions of the publication. SPIE uses a seven-digit CID article numbering system structured as follows:

- The first five digits correspond to the SPIE volume number.

- The last two digits indicate publication order within the volume using a Base 36 numbering system employing both numerals and letters. These two-number sets start with $00,01,02,03$, 04, 05, 06, 07, 08, 09, OA, OB ... OZ, followed by 10-1Z, 20-2Z, etc. The CID Number appears on each page of the manuscript. 


\title{
Contents
}

\author{
xi Authors \\ ix Conference Committee \\ xi 2018 Medical Imaging Award Recipients
}

\section{DEEP LEARNING}

1057603 Automatic slice segmentation of intraoperative transrectal ultrasound images using convolutional neural networks [10576-2]

1057604 Generative adversarial networks for specular highlight removal in endoscopic images [10576-3]

1057605 Tumor margin classification of head and neck cancer using hyperspectral imaging and convolutional neural networks [10576-4]

1057606 Inverse biomechanical modeling of the tongue via machine learning and synthetic training data [10576-97]

1057607 Cine cardiac MRI slice misalignment correction towards full 3D left ventricle segmentation [10576-6]

\section{KEYNOTE AND MEDICAL ROBOTICS}

1057609 Toward image-guided partial nephrectomy with the da Vinci robot: exploring surface acquisition methods for intraoperative re-registration [10576-8]

10576 OA Technical note: feasibility of photoacoustic guided hysterectomies with the da Vinci robot [10576-9]

\section{IMAGE REGISTRATION}

10576 OC Clustered iterative sub-atlas registration for improved deformable registration using statistical shape models [10576-11]

10576 OD Technical note: nonrigid registration for laparoscopic liver surgery using sparse intraoperative data [10576-12]

10576 OE Real-time image-based 3D-2D registration for ultrasound-guided spinal interventions [10576-13] 
10576 OF Influence of 4D CT motion artifacts on correspondence model-based 4D dose accumulation [10576-14]

10576 OG Deformable registration of radiation isodose lines to delayed contrast-enhanced magnetic resonance images for assessment of myocardial lesion formation following proton beam therapy [10576-15]

WORKSHOP: SELECTED PAPERS FROM THE JOURNAL OF MEDICAL IMAGING SPECIAL ISSUE

$10576 \mathrm{OH} \quad$ Technical note: on-the-fly augmented reality for orthopaedic surgery using a multi-modal fiducial [10576-102]

10576 Ol Technical note: probabilistic visual and electromagnetic data fusion for robust drift-free sequential mosaicking: application to fetoscopy [10576-103]

10576 OJ Technical note: an augmented reality system for total hip arthroplasty [10576-104]

10576 OK Technical note: automatic segmentation method of pelvic floor levator hiatus in ultrasound using a self-normalising neural network [10576-105]

$10576 \mathrm{OL} \quad$ Technical note: known-component registration for robotic drill guide positioning [10576-106]

10576 OM Technical note: design and validation of an open-source library of dynamic reference frames for research and education in optical tracking [10576-107]

10576 ON Technical note: on cardiac ablation lesion visualization for image-guided therapy monitoring [10576-109]

$1057600 \quad$ Technical note: a radiomic signature of infiltration in peritumoral edema predicts subsequent recurrence in glioblastoma [10576-110]

\section{NEUROLOGICAL PROCEDURES AND TECHNOLOGIES}

10576 OP Model-based correction for brain shift in deep brain stimulation burr hole procedures: a comparison using interventional magnetic resonance imaging [10576-16]

$105760 Q \quad$ Resection-induced brain-shift compensation using vessel-based methods [10576-17]

10576 OR X-ray image guidance workflow development for in-vivo aneurysm treatment using a new retrievable asymmetric flow diverter (RAFD) [10576-18]

10576 OS Image updating for brain deformation compensation: cross-validation with intraoperative ultrasound [10576-19]

10576 OT Neurosurgical burr hole placement using the Microsoft HoloLens [10576-20] 
ULTRASOUND IMAGING AND DETECTION METHODS

10576 OU 3D ultrasound guidance system for permanent breast seed implantation: integrated system performance and phantom procedure [10576-21]

10576 OV Feature study on catheter detection in three-dimensional ultrasound [10576-22]

10576 OW Coherent needle detection in ultrasound volumes using 3D conditional random fields [10576-23]

10576 OX Compliant joint echogenicity in ultrasound images: towards highly visible steerable needles [10576-24]

10576 OY Real-time transverse process detection in ultrasound [10576-25]

$10576 \mathrm{OZ} \quad$ Visual aid for identifying vertebral landmarks in ultrasound [10576-26]

ENHANCED REALITY, SIMULATION, AND PLANNING

$1057610 \quad$ Assisted needle guidance using smart see-through glasses [10576-27]

$1057611 \quad$ Exploration using holographic hands as a modality for skills training in medicine [10576-28]

$1057612 \quad$ High fidelity virtual reality orthognathic surgery simulator [10576-29]

1057613 Augmented reality needle ablation guidance tool for irreversible electroporation in the pancreas [10576-30]

$1057614 \quad$ Augmented reality assistance in training needle insertions of different levels of difficulty [10576-100]

SEGMENTATION AND MODELING

1057615 Automated segmentation and radiomic characterization of visceral fat on bowel MRIs for Crohn's disease [10576-32]

1057616 A semiautomatic algorithm for three-dimensional segmentation of the prostate on CT images using shape and local texture characteristics [10576-33]

1057617 Auto-contouring via automatic anatomy recognition of organs at risk in head and neck cancer on CT images [10576-34]

1057618 Optimal multimodal virtual bronchoscopy for convex-probe endobronchial ultrasound [10576-35] 
$1057619 \quad$ Machine learning-based colon deformation estimation method for colonoscope tracking [10576-36]

\section{CARDIAC AND LUNG IMAGING AND TRACKING}

10576 1A A real-time system for prosthetic valve tracking [10576-37]

10576 1B Determining in-silico left ventricular contraction force of myocardial infarct tissue using a composite material model [10576-38]

10576 1C A machine learning approach for biomechanics-based tracking of lung tumor during external beam radiation therapy [10576-39]

10576 1D Lung deformation between preoperative CT and intraoperative CBCT for thoracoscopic surgery: a case study [10576-40]

10576 1E Regional lung ventilation estimation based on supervoxel tracking [10576-41]

\section{INTRAOPERATIVE IMAGING AND TECHNOLOGIES}

10576 1F Trackerless surgical image-guided system design using an interactive extension of 3D Slicer [10576-42]

$105761 G$ Advanced image registration and reconstruction using the O-Arm system: dose reduction, image quality, and guidance using known-component models [10576-43]

$10576 \mathrm{1H} \quad$ A system for automatic monitoring of surgical instruments and dynamic non-rigid surface deformations in breast cancer surgery [10576-44]

$1057611 \quad$ Intraoperative deformation during laryngoscopy of irradiated and non-irradiated patients [10576-45]

\section{ABDOMINAL IMAGING AND GUIDANCE TECHNOLOGIES}

$10576 \mathrm{LL} \quad$ Needle deflection in thermal ablation procedures of liver tumors: a CT image analysis [10576-48]

$105761 \mathrm{M} \quad$ Atomic force stiffness imaging: capturing differences in mechanical properties to identify and localize areas of prostate cancer tissue [10576-49]

10576 iN Automatic definition of surgical trajectories and acceptance window in pelvic trauma surgery using deformable registration [10576-50]

1057610 Intra-operative $360^{\circ}$ 3D transvaginal ultrasound guidance during high-dose-rate interstitial gynecologic brachytherapy needle placement (Young Scientist Award) [10576-51] 
10576 1P Ring navigation: an ultrasound-guided technique using real-time motion compensation for prostate biopsies [10576-52]

\section{VALIDATION, SIMULATION, AND 3D PRINTING}

$105761 Q \quad$ Using water-soluble additive manufacturing for cheap and soft silicon organ models [10576-57]

10576 IR PedBot: robotically assisted ankle robot and video game for children with neuromuscular disorders [10576-54]

10576 is A mold design for creating low-cost patient specific models with complex anatomy [10576-55]

10576 1T 3D tissue mimicking biophantoms for ultrasound imaging: bioprinting and image analysis [10576-56]

$105761 \mathrm{U} \quad$ Validation of cochlear implant electrode localization techniques [10576-53]

\section{POSTER SESSION}

$105761 \mathrm{~V} \quad$ Vessel layer separation in x-ray angiograms with fully convolutional network [10576-5]

10576 1X Geometric modeling of the aortic inner and outer vessel wall from CTA for aortic dissection analysis [10576-58]

$1057619 \quad$ Develop and validate a finite element method model for deformation matching of laparoscopic gastrectomy navigation [10576-59]

$1057612 \quad$ Bayesian delineation framework of clinical target volumes for prostate cancer radiotherapy using an anatomical-features-based machine learning technique [10576-60]

1057620 Real-time workflow detection using webcam video for providing real-time feedback in central venous catheterization training [10576-61]

$1057621 \quad$ Control of real-time MRI with a 3D controller during radiofrequency ablation [10576-62]

1057622 In vivo reconstruction of coronary artery and bioresorbable stents from intracoronary optical coherence tomography [10576-63]

1057623 Automated location detection of injection site for preclinical stereotactic neurosurgery through fully convolutional network [10576-64]

1057624 Pre- to post-operative CT image registration to estimate cortical shift for image updating in deep brain stimulation [10576-65] 
1057625 A learning curve analysis of ultrasound-guided in-plane and out-of-plane vascular access training with Perk Tutor [10576-66]

1057626 Clinical feasibility of $\mathbf{x}$-ray based pose estimation of a transthoracic echo probe using attached fiducials [10576-67]

1057627 Towards webcam-based tracking for interventional navigation [10576-68]

$1057628 \quad$ HoloLens in suturing training [10576-69]

10576 2C Architectural analysis on dynamic MRI to study thoracic insufficiency syndrome [10576-73]

10576 2D Improvement of liver ablation treatment for colorectal liver metastases [10576-74]

$105762 \mathrm{E} \quad$ Hippotherapy simulator for children with cerebral palsy [10576-75]

$105762 \mathrm{~F} \quad$ Quantitative assessment of cardiac motion using multiphase computed tomography imaging with application to cardiac ablation therapy [10576-76]

$105762 \mathrm{H} \quad$ Liver surface reconstruction for image guided surgery [10576-78]

$1057621 \quad$ Fusing acoustic and optical sensing for needle tracking with ultrasound [10576-79]

$105762 \mathrm{~J} \quad$ Treatment plan library based on population shape analysis for cervical adaptive radiotherapy [10576-81]

$105762 \mathrm{~K} \quad$ Ultrathin and flexible 4-channel scope for guiding surgical resections using a near-infrared fluorescence molecular probe for cancer [10576-82]

$105762 \mathrm{~L} \quad$ Ultrasound imaging of the posterior skull for neurosurgical registration [10576-83]

$105762 \mathrm{M}$ Development of an augmented reality approach to mammographic training: overcoming some real world challenges [10576-84]

$105762 \mathrm{~N} \quad$ Image quality and segmentation [10576-85]

1057620 Distant pulse oximetry based on skin region extraction and multi-spectral measurement [10576-86]

$105762 \mathrm{P} \quad$ Tracking of liver vessel bifurcations in $3 \mathrm{D}+\mathrm{t}$ ultrasound by subsequent approximations of a rigid shape model [10576-87]

$105762 \mathrm{Q}$ Precision blood flow measurements in vascular networks with conservation constraints [10576-88]

$105762 R \quad$ Osteotomy planner: an open-source tool for osteotomy simulation [10576-89]

1057625 In vivo imaging of radiopaque resorbable inferior vena cava filter infused with gold nanoparticles [10576-90] 
$105762 \mathrm{U}$ Micromechanics based modelling of in-vivo respiratory motion of the diaphragm muscle with the incorporation of optimized z-disks mechanics [10576-92]

10576 2V Cone beam tomosynthesis fluoroscopy: a new approach to 3D image guidance [10576-93]

$105762 \mathrm{~W}$ Surgical skill level assessment using automatic feature extraction methods [10576-94]

10576 2X Bundling 3D- and 2D-based registration of MRI to $\mathrm{x}$-ray breast tomosynthesis [10576-95]

$105762 Y \quad T$ Towards robust needle segmentation and tracking in pediatric endoscopic surgery [10576-96]

$105762 Z$ CT-ultrasound deformable registration for PET-determined prostate brachytherapy (Cum Laude Poster Award) [10576-99]

1057630 ProjectAlign: a real-time ultrasound guidance system for spinal midline detection during epidural needle placement [10576-101] 
Proc. of SPIE Vol. 10576 1057601-10 Downloaded From: https://www.spiedigitallibrary.org/conference-proceedings-of-spie on 26 Apr 2023
Terms of Use: https://www.spiedigitallibrary.org/terms-of-use 


\section{Authors}

Numbers in the index correspond to the last two digits of the seven-digit citation identifier (CID) article numbering system used in Proceedings of SPIE. The first five digits reflect the volume number. Base 36 numbering is employed for the last two digits and indicates the order of articles within the volume. Numbers start with 00, 01, 02, 03, 04, 05, 06, 07, 08, 09, OA, OB...0Z, followed by 10-12, 20-2Z, etc.

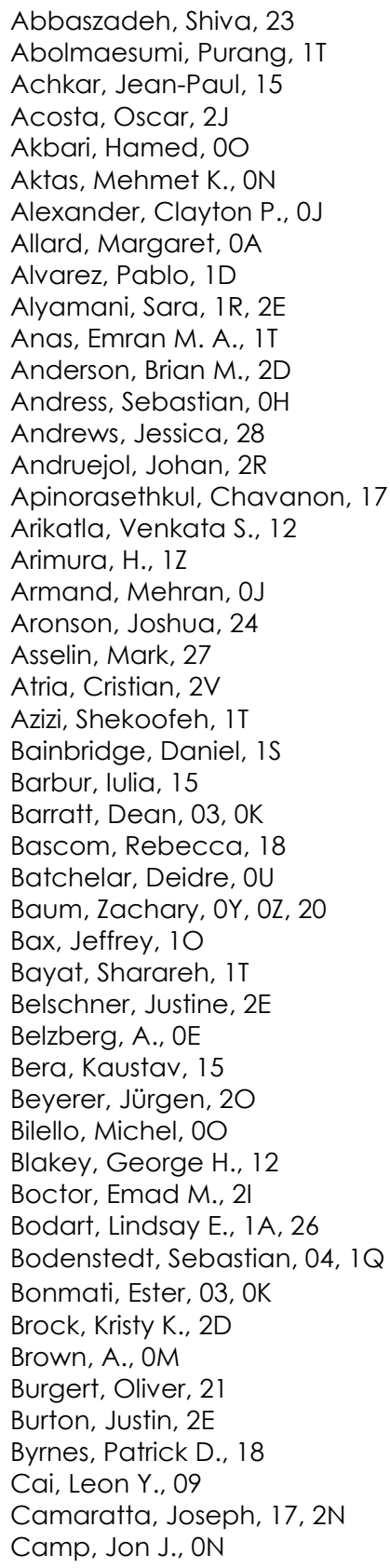

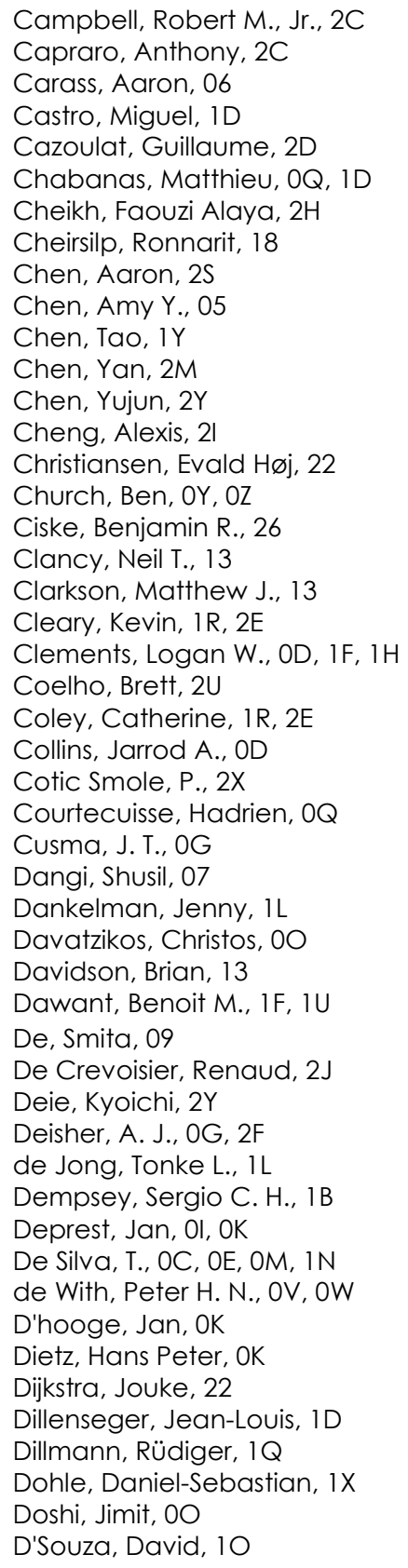


Eggers, Mitch, 2S

Eigen, Katharina, $1 \mathrm{X}$

Elle, Ole Jakob, $2 \mathrm{H}$

Emmanuel, Steve, 28

Enquobahrie, Andinet, 12, 2R

Ershad, Marzieh, 2W

Essmann, Clara, 1M

Evans, Sally, 1R, 2E

Fan, Xiaoyao, OS, 24

Fei, Baowei, 05, 16

Fei, Zhenyu, 22

Fenster, Aaron, 0U, 10, $1 \mathrm{P}$

Ferguson, James M., 09

Fichtinger, Gabor, OT, OY, 0Z, 11, 14, 20, 25, 27, 28 , $2 \mathrm{~L}$

Fiocchi, Claudio, 15

Fooladi Talari, Hadi, 1R, 2E

Fotouhi, Javad, $\mathrm{OH}, \mathrm{OJ}$

Freeman, Alex, $1 \mathrm{M}$

Fuerst, Bernhard, OJ

Funke, Isabel, 04, 1Q

Furukawa, Kazuhiro, 19

Gaede, Stewart, $1 \mathrm{C}$

Gale, Alastair G., 2M

Gardi, Lori, 1P

Gaver, Tobias, OF

Geevarghese, Sunil K., OD

Gern, Markus, 1Q

Ghavami, Nooshin, 03

Ghazvinian Zanjani, Farhad, OW

Gibson, Eli, 03

Gillies, Derek J., 1P

Girard, Emily J., 2K

Gleeson, Fergus V., 1E

Gobeli, Maxime, 2J

Goerres, J., OC, 1N

Gomez, Arnold D., 06

Goodale, Sarah E., $1 \mathrm{H}$

Goodworth, Adam, 2E

Goto, Hidemi, 19

Grau, Vicente, 1E

Griffith, Christopher C., 05

Gunka, Vit, 30

Guo, Rongrong, 16

Gupta, Rishi, 15

Gupta, Sanjay, 2D

Gurusamy, Kurinchi, 13

Haemmerich, Dieter, ON

Haigron, Pascal, 2J

Halicek, Martin, 05, 16

Halter, Ryan J., 11

Han, R., OC, OE, IN

Hao, Haidong, IV

Haq, Hassan, 28

Harada, Kanako, $2 Y$

Hasnain, A. C., 2F

Hawkes, David J., 13

Hayashi, Yuichiro, IY

Heiselman, Jon S., OD

Helm, P., $1 G$
Herman, M. G., OG

Herrell, S. Duke, III, 09

Herrmann, Christian, 20

Higgins, William E., 18

Hilts, Michelle, OU

Hirooka, Yoshiki, 19

Hirose, T., 12

Hisey, Rebecca, 20, 25

Hoffman, Carson, 2Q

Hoffmann, Rüdiger, 21

Hohmann, S., OG, 2F

Holck, Emil N., 22

Holden, Matthew S., OT, 1 1, 20, 25

Holm, Niels R., 22

Holmes, David R., III, ON

Honda, H., $1 \mathrm{Z}$

Hopp, T., $2 X$

Horvath, Sam, 2R

Howes, Daniel W., 20

Hu, Yipeng, 03, OK

Huang, David T., ON

Huang, Steven, $2 S$

Iglesias, Juan Eugenio, 01

Ionita, Ciprian N., OR

Jacobsen, Megan C., $2 S$

Jacobson, Matt, OC, OE, IN

James, Paul, 17

Janatka, Mirek, 13

Jarnagin, William R., OD

Jiang, Yang, 2K

Jiang, Zhengang, $1 Y$

Johnson, Alex, $\mathrm{OH}, \mathrm{OJ}$

Kaaniche, Mounir, $2 \mathrm{H}$

Kaiser, C., 2X

Kamali, Shahrokh, OY

Karami, Elham, 1C

Kay, Marsha, 15

Kenngott, Hannes, 1Q

Keri, Zsuzsanna, 20, 25, 28

Ketcha, M., OC, OE, 1N

Khanuja, Harpal, OJ

Kingham, T. Peter, OD

Kitasaka, Takayuki, 19

Kleinszig, G., OC, OE, IN

Klink, Camiel, $1 \mathrm{~L}$

Kolen, Alexander F., OV

Konishi, H., OG, 2F

Korsten, Hendrikus H. M., OW

Kovelman, Staci, $1 \mathrm{R}$

Krammer, J., $2 X$

Kronreif, Gernot, 2L

Kruse, J. J., OG

Kuhlengel, Trevor K., 18

Kurose, Yusuke, 2 Y

Kurowski, Jacob, 15

Kuzhagaliyev, Timur, 13

Labadie, Robert F., $1 \mathrm{U}$

Laeseke, Paul, 2Q

Lang $\varnothing$, Thomas, OX

Larson, Paul S., OP 
Lasso, Andras, OT, OY, 0Z, 11, 27, 28, 2L Last, Lisa, 2V

Lediju Bell, Muyinatu A., OA

Lee, Junghoon, $2 Z$

Lee, Patrick, $2 S$

Lee, Sing Chun, 0J

Lee, Ting-Yim, $1 \mathrm{C}$

Lehmann, H. I., OG

Le Lann, Florian, $\mathrm{OQ}$

Leseur, Julie, 2J

Lessoway, Victoria A., 30

Leung, Eric, 10

Leung, Regina, 11, 14

Levy, Ron, OT

Li, Chen, OS, 24

Li, Guoxin, $1 Y$

Li, Ming, 10

Li, Yingguang, 22

Lia, Hillary, 25, 28

Lin, Ethan Y., 2D

Linguraru, Marius George, 2R

Linte, Cristian A., 07, ON

Little, James $\vee ., 05$

Liu, Zheng, 23

Lu, Linfeng, $2 S$

Ludig, Kristian, 28

Lukens, John, 17

Luo, Ma, OP, 1F, $1 \mathrm{H}$

Lustig, Robert, 00

Ma, Hua, IV

Ma, Ling, 16

Maier, Andreas, $1 \mathrm{X}$

Majewicz Fey, Ann, 2W

Manbachi, A., OM

Marinho, Murilo M., $2 Y$

Martin, Alastair J., OP

Martinez, Jonathan, $2 S$

Massey, Simon, 30

Master, Viraj, 16

Matin, Tahreema, $1 \mathrm{E}$

McArthur, Mark, 2S

McCallum, Caitlin, 20

McDonough, Joseph, 2C

McGraw, Robert, 14

McLaughlin, David J., 17, 2N

Melancon, Adam, 25

Melancon, Marites P., $2 S$

Mercado, Ashley, IP

Meszoely, Ingrid M., $1 \mathrm{H}$

Metzler, Jürgen, 20

Michael, Justin, OU

Miga, Michael I., OD, OP, 1F, 1H

Mihailidis, Dimitris, 17

Mihajlovic, Nenad, OW

Mistretta, Charles A., 2Q

Mitchell, Christopher H., 25

Mitsuishi, Mamoru, $2 Y$

Miyahara, Ryoji, 19

Moelker, Adriaan, $1 \mathrm{~L}$

Mohamed, Tamer, $1 T$
Monfaredi, Reza, 1R, 2E

Moore, Caroline, 03

Moore, John, is

Mori, Kensaku, 19, 1Y

Morin, Evelyn, OT

Morin, Fanny, $\mathrm{OQ}$

Morozova, Olga, 2E

Morton, Daniel, OU

Moult, Eric, 14

Mousavi, Parvin, $1 T$

Müller-Stich, Beat Peter, $1 Q$

Nakazawa, Atsushi, $2 Y$

Narasimhan, Saramati, OP

Navab, Nassir, OH, OJ, 19

Newman, L. K., OG, $2 F$

Ng, Gary C., OW

Nguyen, Tung, 12

Nieh, Peter, 16

Ninomiya, K., 12

Noble, Jack H., $1 \mathrm{U}$

Noo, Frederic, 2V

Oda, Masahiro, 19, 1 Y

Odhner, Dewey, 17

Odisio, Bruno C., 2D

Oh, Philip, 21

Ohga, S., 1 Z

Olson, Jonathan D., OS

Osgood, Greg, OC, OH, 0J, 1G, IN

Ourselin, Sebastien, 이

Overhoff, Heinrich M., 2P

Packard, Nathan, 2V

Packer, Douglas L., 0G, ON, 2F

Pakiam, Fiona, 2K

Palombi, Olivier, $0 Q$

Paniagua, Beatriz, 12, 2R

Papiez, Bartłomiej W., IE

Parker, K. D., OG, 2F

Patel, Mihir, 05

Paulin, Gregory, 28

Paulsen, Keith D., OS, 24

Pawar, Vijay M., $1 M$

Payan, Yohan, OQ, 1D

Paydarfar, Joseph A., 11

Pednekar, Gargi V., 17, 2N

Pergami, Paola, $1 \mathrm{R}$

Peter, Loic, 01

Peters, Terry, is

Pinter, Csaba, oy

Podgorsak, Alexander, OR

Porras, Antonio R., 2R

Pourtaherian, Arash, OV, OW

Prince, Jerry L., 06

Pylatiuk, Christian, 1Q

Qiao, Yang, 2S

Rae, Emily, OT

Rajaram, Ajay, $1 \mathrm{~T}$

Ramchandran, $\mathrm{V}$., OL

Ramsay, B., OC, IN

Rathore, Saima, 00

Raval, Amish N., 1A, 26 
Reed, Alexander, 09

Rege, Robert, 2W

Reiber, Johan H. C., 22

Reichard, Daniel, $1 Q$

Reinertsen, Ingerid, $O Q$

Rettmann, Maryam E., OG, ON, 2F

Richey, Winona L., $1 \mathrm{H}$

Riediger, Carina, 04

Rigaud, Bastien, 2J

Roberts, David W., OS

Rodell, Rachael, 03

Rodgers, Jessica R., OU, 10

Rohling, Robert N., 30

Rouzé, Simon, 1D

Rozycki, Martin, 00

Ruiter, N. V., $2 X$

Saavedra, Sandra, $2 \mathrm{E}$

Salvador, Tyler, 1R, 2E

Samani, Abbas, 1B, 1C, 2 U

Sánchez-Margallo, Juan A., OX

Sargent, Derek, 14

Sasahara, M., $1 \mathrm{Z}$

Sasaki, T., $1 \mathrm{Z}$

Schaefer, Gerald, $2 M$

Schafer, Sebastian, 1A, 2Q

Schlenger, Christopher, $\mathrm{OZ}$

Schnabel, Julia A., $1 \mathrm{E}$

Schuster, David M., 16

Sciubba, D. M., OE

Seibel, Eric J., 2K

Shahedi, Maysam, 16

Shakir, Dzhoshkun I., 01

Shammo, Geraldine, 17

Shan, Caifeng, OV

Shaughnessy, Gabe, $2 Q$

Shepard, Lauren M., OR

Sheth, Niral, OC, OE, 1N

Shi, Weili, $1 Y$

Shubert, Joshua, OA

Shukla, Gaurav, 00

Siddiqui, Adnan, OR

Siebold, Michael, 09

Siewerdsen, J. H., OC, OE, OL, OM, 1G, IN

Simon, Antoine, 2J

Simone, Charles B., II, 17, 2N

Simpson, Amber L., OD

Sindhwani, Nikhil, OK

Singhana, Burapol, $2 S$

Sommer, Kelsey N., OR

Song, Daniel Y., $2 Z$

Song, Jie, 2C

Sothmann, Thilo, OF

Speidel, Michael A., 1A, 26

Speidel, Stefanie, 04, 1Q

Stayman, J. W., $1 G$

Stone, Maureen L., 06

Stoyanov, Danail, 0l, 13, 1M

Strother, Charles M., 2Q

Sühling, Michael, $1 \mathrm{X}$

Surry, Kathleen, 10
Suzuki, A., OG, 2F

Szmul, Adam, 1E

Tabrizi, Pooneh R., 1R, 2E

Tang, Qiang, $2 \mathrm{M}$

Tang, Xikai, OW

Tasciotti, Ennio, $2 \mathrm{~S}$

Taylor, Giacomo, 0J

Taylor, Russell H., OJ

Tchaka, Kevin, 13

Tella-Amo, Marcel, ol

Tessier, David, IP

Thawani, Rajat, 15

Theodore, N., $1 G$

Thompson, Reid C., IF

Tian, Li, $2 S$

Toews, Alexander R., 30

Tolpadi, Aniket A., 06

Tong, Yubing, 17, 2C, 2N

Torigian, Drew A., 17, 2C, 2N

Toth, Jennifer, 18

Travers, Bryan, OY

Tu, Shengxian, 22

Tyagi, Mohit, 12

Udupa, Jayaram K., 17, 2C, 2N

Umezu, Y., $1 Z$

Unberath, Mathias, $\mathrm{OH}, \mathrm{OJ}$

Underwood, Grace, 2L

Uneri, A., OC, OE, OL, OM, 1G, IN

Ungi, Tamas, OY, OZ, 14, 20, 25, 27, 2L

van de Berg, Nick J., OX

van den Dobbelsteen, John J., OX, $1 \mathrm{~L}$

van Walsum, Theo, $1 \mathrm{~V}$

Vasconcelos, Francisco, 13

Vassallo, Reid, $1 \mathrm{~S}$

Velker, Vikram, 10

Venkataraman, Ashwin, OR

Vercauteren, Tom, Ol, OK

Vijayan, Rohan, $1 \mathrm{~F}$

Viswanath, Satish, 15

Vogt, S., OC, OE, IN

Wagner, Martin, 1A, 26

Walus, Konrad, IT

Wang, Congcong, $2 \mathrm{H}$

Wang, S., OG, 2F

Wang, $X u, 05$

Webster, Robert J., III, 09

Wei, Guodong, 1 Y

Weidert, Simon, $\mathrm{OH}$

Weis, Jared A., OD

Weitz, Jürgen, 04

Wels, Michael, $1 \mathrm{X}$

Werner, René, OF

White, Ray, 12

Willersinn, Dieter, 20

Williaume, Danièle, $2 \mathrm{~J}$

Winkler, Alexander, $\mathrm{OH}$

Wood, Brad J., 10

Wu, Caiyun, 2C

Wu, Hemmings, 23

Wu, Xiaotian, 11 
Wu, Xingyu, 17, 2N

Xia, Sean, 25

Xiao, Liang, 2C

$\mathrm{Xu}$, Sheng, 10

Yang, Hongxu, OV

Yang, Xiaochen, $1 \mathrm{~F}$

Yaniv, Ziv, 07

Yeo, Caitlin T., 14, 28

Yi, Nelson, 28

Yi, T., OL

Yu, Kevin, $\mathrm{OH}$

Zang, Xiaonan, 18

Zevin, Boris, 11

Zhang, Bofeng, 2

Zhang, Guoyi, 16

Zhang, X., OE, $1 \mathrm{G}$

Zhao, Yiyuan, $1 \mathrm{U}$

Zinger, Svitlana, OW

Zurawka, Vanessa, 21

Proc. of SPIE Vol. 10576 1057601-15

Downloaded From: https://www.spiedigitallibrary.org/conference-proceedings-of-spie on 26 Apr 2023 Terms of Use: https://www.spiedigitallibrary.org/terms-of-use 
Proc. of SPIE Vol. 10576 1057601-16

Downloaded From: https://www.spiedigitallibrary.org/conference-proceedings-of-spie on 26 Apr 2023 Terms of Use: https://www.spiedigitallibrary.org/terms-of-use 


\section{Conference Committee}

Symposium Chairs

Leonard Berliner, Weill Cornell Medical College (United States) and New York Presbyterian - Brooklyn Methodist Hospital (United States)

Ronald M. Summers, National Institutes of Health (United States)

Conference Chairs

Baowei Fei, Emory University (United States)

Robert J. Webster III, Vanderbilt University (United States)

Conference Program Committee

Purang Abolmaesumi, The University of British Columbia (Canada)

Wolfgang Birkfellner, Medizinische Universität Wien (Austria)

Elvis C. S. Chen, Robarts Research Institute (Canada)

Sandrine de Ribaupierre, Western University (Canada)

Gabor Fichtinger, Queen's University (Canada)

George J. Grevera, Saint Joseph's University (United States)

David Hawkes, University College London (United Kingdom)

David R. Haynor, University of Washington (United States)

William E. Higgins, The Pennsylvania State University (United States)

David R. Holmes III, Mayo Clinic (United States)

Pierre Jannin, Université de Rennes 1 (France)

David M. Kwartowitz, Grand Canyon University (United States)

Cristian A. Linte, Rochester Institute of Technology (United States)

Lena Maier-Hein, Deutsches Krebsforschungszentrum (Germany)

Michael I. Miga, Vanderbilt University (United States)

Kensaku Mori, Nagoya University (Japan)

Parvin Mousavi, Queen's University (Canada)

Jack H. Noble, Vanderbilt University (United States)

Maryam E. Rettmann, Mayo Clinic (United States)

Frank Sauer, Siemens Healthineers (United States)

Eric J. Seibel, University of Washington (United States)

Guy Shechter, Philips Healthcare (United States)

Jeffrey H. Siewerdsen, Johns Hopkins University (United States)

Amber L. Simpson, Memorial Sloan-Kettering Cancer Center

(United States)

Stefanie Speidel, Karlsruher Institut für Technologie (Germany)

Andrew D. Wiles, Northern Digital Inc. (Canada)

Ivo Wolf, Hochschule Mannheim (Germany)

Ziv R. Yaniv, National Library of Medicine (United States) 
Deep Learning

Cristian A. Linte, Rochester Institute of Technology (United States)

Terry Peters, Robarts Research Institute (United States)

Keynote and Medical Robotics

Baowei Fei, Emory University (United States) and Georgia Institute of Technology (United States)

Robert J. Webster III, Vanderbilt University (United States)

Image Registration

William E. Higgins, The Pennsylvania State University (United States)

Neurological Procedures and Technologies

Ziv Yaniv, National Library of Medicine (United States)

Gabor Fichtinger, Queen's University (Canada)

Ultrasound Imaging and Detection Methods

David M. Kwartowitz, Grand Canyon University (United States)

Yu-Ping Wang, Tulane University (United States)

Enhanced Reality, Simulation, and Planning

Elvis C. S. Chen, Robarts Research Institute (Canada)

David R. Holmes III, Mayo Clinic (United States)

Segmentation and Modeling

David R. Holmes III, Mayo Clinic (United States)

David R. Haynor, University of Washington (United States)

Cardiac and Lung Imaging and Tracking

Maryam E. Rettmann, Mayo Clinic (United States)

Amber L. Simpson, Memorial Sloan-Kettering Cancer Center (United States)

Intraoperative Imaging and Technologies

Purang Abolmaesumi, The University of British Columbia (Canada)

Ryan J. Halter, Thayer School of Engineering at Dartmouth (United States)

Abdominal Imaging and Guidance Technologies

Kensaku Mori, Nagoya University (Japan)

Jeffrey H. Siewerdsen, Johns Hopkins University (United States)

Validation, Simulation, and 3D Printing

Jack H. Noble, Vanderbilt University (United States)

Ivo Wolf, Hochschule Mannheim (Germany) 


\section{Medical Imaging Award Recipients}

\section{Robert F. Wagner Best Student Paper Award}

Robert F. Wagner was an active scientist in the SPIE Medical Imaging meeting, starting with the first meeting in 1972 and continuing throughout his career. He ensured that the $\mathrm{BRH}$, and subsequently the $\mathrm{CDRH}$, was a sponsor for the early and subsequent Medical Imaging meetings, helping to launch and ensure the historical success of the meeting. The Robert F. Wagner All-Conference Best Student Paper Award (established 2014) is acknowledgment of his many important contributions to the Medical Imaging meeting and his many important advances to the field of medical imaging.

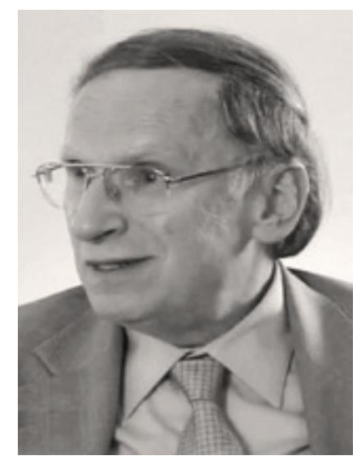

This award is co-sponsored by:

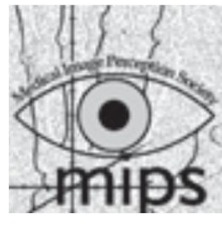

The Medical Image Perception Society

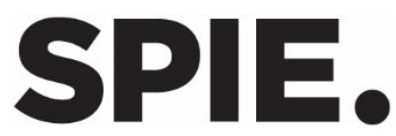

\section{Recipients:}

First Place: Dynamic beam filtering for miscentered patients (10573-29)

Andrew Mao, William Shyr, Grace J. Gang, J. Webster Stayman, Johns Hopkins Univ. (United States)

Second Place: Tumor margin classification of head and neck cancer using hyperspectral imaging and convolutional neural networks (10576-4)

Martin Halicek, Georgia Institute of Technology (United States) and Augusta Univ. (United States); James V. Little, Xu Wang, Emory Univ. School of Medicine (United States); Mihir Patel, Emory Univ. School of Medicine (United States) and The Winship Cancer Institute of Emory Univ. (United States); Christopher C. Griffith, Emory Univ. School of Medicine (United States); Amy Y. Chen, Emory Univ. School of Medicine (United States) and The Winship Cancer Institute of Emory Univ. (United States); Baowei Fei, Georgia Institute of Technology \& Emory Univ. (United States) and The Winship Cancer Institute of Emory Univ. (United States)

\section{Conference Awards}

\section{Image-Guided Procedures, Robotic Interventions, and Modeling Young Scientist Awards sponsored by Siemens Healthineers}

Winner: Paper 10576-51, "Intra-operative 360 3D transvaginal ultrasound guidance during high-dose-rate interstitial gynecologic brachytherapy needle placement"

J. R. Rodgers, Western Univ. (Canada) and Robarts Research Institute (Canada); J. Bax, Robarts Research Institute (Canada); V. Velker, K. Surry, D. D'Souza, London Regional Cancer Program (Canada); E. Leung, Odette Cancer Ctr. (Canada); A. Fenster, Western Univ. (Canada) and Robarts Research Institute (Canada) 
Runner-up: Paper 10576-53, "Validation of cochlear implant electrode localization techniques"

Y. Zhao, Vanderbilt Univ. (United States); R. F. Labadie, Vanderbilt Univ. Medical Ctr. (United States); B. M. Dawant, J. H. Noble Sr., Vanderbilt Univ. (United States)

Runner-up: Paper 10576-56, "3D tissue mimicking biophantoms for ultrasound imaging: bioprinting and image analysis"

S. Azizi, S. Bayat, The Univ. of British Columbia (Canada); A. Rajaram, Queen's Univ. (Canada); E. Anas, Johns Hopkins Univ. (United States); T. Mohamed, K. Walus, Aspect Biosystems Ltd. (Canada); P. Abolmaesumi, The Univ. of British Columbia (Canada); P. Mousavi, Queen's Univ. (Canada)

Image-Guided Procedures, Robotic Interventions, and Modeling Poster Presentation Awards sponsored by Northern Digital Imaging

Cum Laude: Paper 10576-99, "CT-ultrasound deformable registration for PET-

determined prostate brachytherapy"

J. Lee, D. Y. Song, Johns Hopkins Univ. (United States)

Honorable Mention: Paper 10576-61, "Real-time workflow detection using webcam video for providing real-time feedback in central venous catheterization training" R. Hisey, T. Ungi, M. Holden, Z. Baum, Z. Keri, Queen's Univ. (Canada); C. McCallum, D. W. Howes, Kingston General Hospital (Canada); G. Fichtinger, Queen's Univ. (Canada)

Honorable Mention: Paper 10576-86, "Distant pulse oximetry based on skin region extraction and multi-spectral measurement"

C. Herrmann, Fraunhofer-Institut für Optronik, Systemtechnik und Bildauswertung (Germany) and Karlsruhe Institute of Technology (Germany); J. Metzler, D. Willersinn, Fraunhofer-Institut für Optronik, Systemtechnik und Bildauswertung (Germany); J. Beyerer, Fraunhofer-Institut für Optronik, Systemtechnik und Bildauswertung (Germany) and Karlsruhe Institute of Technology (Germany) 